\title{
Measurement of Modal Damping Factor to Monitor Bone Integrity and Osteopenia: A Study on Sheep Trochanters
}

Sofia D. Panteliou ${ }^{1 *}$, Elias Panagiotopoulos ${ }^{2}$, John Varakis ${ }^{2}$, Malvina Orkoula ${ }^{3}$ and Chris Kontoyannis ${ }^{3}$

${ }^{1}$ Department of Mechanical Engineering and Aeronautics, University of Patras, Greece

${ }^{2}$ Medical School, University of Patras, Greece

${ }^{3}$ Department of Pharmacy, University of Patras, and FORTH/ICE-HT, Geece

\begin{abstract}
A new non-destructive method was applied in order to assess bone integrity. The method is based on measurement of bone dynamic characteristics (MDF and QF) by applying vibration excitation in the range of acoustic frequencies, in the form of an acoustic sweep signal. The method has been already applied on metallic structures and composites, including animal bones and has shown the potential to become a valuable assessment tool for monitoring bone structural changes. The measured characteristics are directly related with stress concentration due to discontinuities in the material of the bone, such as changes in porosity due to osteopenia. In turn, stress concentration is known to cause fracture in such materials. In this work, sheep femora were split in cuts and tested to detect changes in MDF - QF, BMD $\left(\mathrm{kg} / \mathrm{m}^{2}\right)$, RS and IEF, without the effect of soft tissue. MDF - QF are shown to be the best indicators and measures of the tendency of the bone to fracture, as compared to the conventional methods (BMD and RS) and to the absolute strength measure (IEF).
\end{abstract}

Keywords: Bone integrity; Modal damping; Osteopenia; Raman spectroscopy; Impact strength; Sheep trochanters

Abbreviations: MDF: Modal Damping Factor; QF: Quality Factor; BMD: Bone Mineral Density; RS: Raman Spectroscopy; IEF - Impact Energy of Fracture; DEXA: Dual Energy X-Ray Absorptiometry; FFT: Fast Fourier Transform

\section{Introduction}

Bone is a composite formed by the mineralization of an organic matrix (largely collagen type I) by the nucleation and growth of a carbonated calcium phosphate mineral (bio apatite), highly resembling hydroxy-apatite, $\mathrm{Ca}_{10}\left(\mathrm{PO}_{4}\right)_{6}(\mathrm{OH})_{2}$, within the matrix. Bioapatite provides the biomechanical properties needed for body support and movement, enabling it also to withstand stresses. Failure of mineralization of newly formed or bone remodelling results in an excess of unmineralized bone matrix (called osteoid tissue) and this comprises the clinical entity of osteomalacia. Main causative mechanisms are vitamin D deficiency and phosphate depletion [1]. In contrary both the decrease in the amount of normally mineralized bone and the disturbance in bone micro architecture (which increases the risk of fractures occurring in the absence of trauma or in response to trivial trauma) is known as osteoporosis [2]. Some clinicians use the generic term osteopenia, which describes decreased mineralised bone mass with no reference in particular bone pathology responsible for the decrease in bone mineral density [1].

Osteopenia may be due to osteoporosis, osteomalacia, primary hyperparathyroidism or malignant diseases, all of which may cause generalized bone loss.

A number of conventional tools have been used for the identification of major bone constituents. RS is based on the irradiation of a sample by laser beam and the observation of the differences between the frequencies of the incident beam and the scattered light. These differences are due to the vibration transitions of the molecules of the sample, thus information on these molecules and their quantity can be extracted. Due to its nature RS is a non-destructive technique for the sample but practically is also a surface technique. Therefore, it is difficult to be applied as a non-invasive technique for molecular analysis of bone. On the other hand RS has unique capabilities such as point-by-point analysis (mapping) of the sample by focusing the laser on the desired area of the sample, non-destructive nature, absence of any sample preparation, very short time needed to record and process a Raman spectrum. RS has been applied recently on bones for identification purposes [3-8], for studying the chemical microstructure of cortical bone [9], for determining the mineral percentage as well as the mineral/matrix ratio depth profile of artificially demineralized bovine femur [10] and sheep femoral heads [11], and in mineralization and biomechanical studies [12].

BMD is recognized as the most important single determinant of fracture risk in populations with bone disease [13,14]. Accordingly, measurement of bone density is currently the mainstay for the diagnosis and monitoring of osteoporosis, osteomalacia or osteopenia in general. However, many other skeletal and extraskeletal factors and conditions may influence the risk of developing a fracture, as in particular, a hip fracture [15]. Therefore, the ability of bone to withstand traumatic insults is the result of both the amount of mineralized tissue per unit of volume (density), and many other factors that are commonly referred to as "bone quality". Loss of trabecular connectivity is considered one of the critical factors that weaken bone strength in osteoporosis [16]. Although data are still limited, this architectural abnormality may independently constitute an important factor for predicting fracture risk [17].

*Corresponding author: Sofia D. Panteliou, Department of Mechanical Engineering and Aeronautics, University of Patras, Greece, E-mail: panteliu@mech.upatras.gr

Received May 07, 2012; Accepted July 20, 2012; Published July 25, 2012

Citation: Panteliou SD, Panagiotopoulos E, Varakis J, Orkoula M, Kontoyannis C (2012) Measurement of Modal Damping Factor to Monitor Bone Integrity and Osteopenia: A Study on Sheep Trochanters. J Bioengineer \& Biomedical Sci 2:118. doi:10.4172/2155-9538.1000118

Copyright: (c) 2012 Panteliou SD, et al. This is an open-access article distributed under the terms of the Creative Commons Attribution License, which permits unrestricted use, distribution, and reproduction in any medium, provided the original author and source are credited. 
In vitro studies have shown that bone strength is correlated not only to mineral content, but also to the modulus of elasticity [18-20] and the natural frequency of bone vibration [21-25]. Thus, transmission techniques based on ultrasound attenuation or velocity, have been developed as a clinical tool [26].

The application of a new analytical-arithmetic and experimental method for measurement and calculation of MDF to assess bone integrity, based on sweeping sound excitation, which is equal or better predictor of bone strength than mineral density, was presented by $[11,27,28]$. This method was also applied in porous isotropic and homogeneous materials with elastic behaviour and gave excellent results $[29,30]$ as well as in cracked structures [31,32] and ceramics [33]. Ultrasound measures the elasticity modulus of the material and uses high frequency sound, while the method reported here measures material damping using excitation in the acoustic range. The calculation of QF based on MDF can in theory be applied in clinical settings to estimate the biomechanical competence of bone, and thus it may be used as a diagnostic tool.

In this work MDF, BMD (with DEXA), RS and IEF were measured on artificially deminineralized cuts taken from sheep femoral heads for correlation purposes. IEF measurements were selected since it is the absolute measure of strength, while MDF, BMD, and RS measurements are only correlating indices. Cutting the femoral heads enabled the composition analysis of the different regions of each head, through RS, before and after demineralization, since, as opposed to previous known works, both the cancellous regions and the cortical part of each bone were available for RS excitation. This course of action was dictated as the next research step to previous works [11,28], where MDF was applied for the first time on intact bones for monitoring the degree of demineralization by immersing the samples in $\mathrm{HCl}$ for different periods or on osteoporotic due to ovariectomy rats, respectively, in order to confirm that the method is equally effective in a localized study.

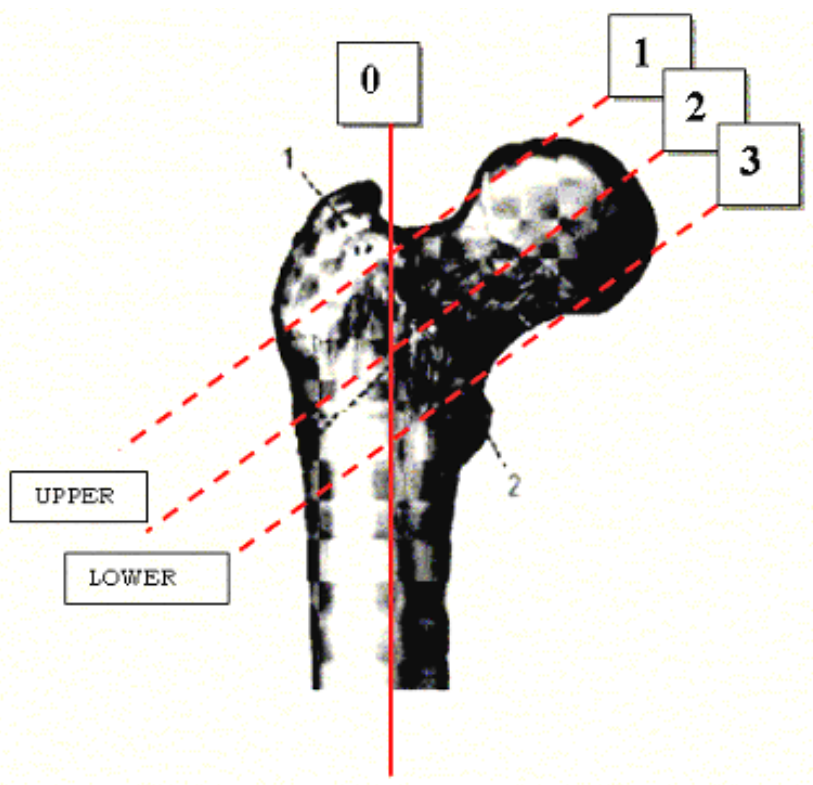

Figure 1: Front view of the bone. Cutting levels.

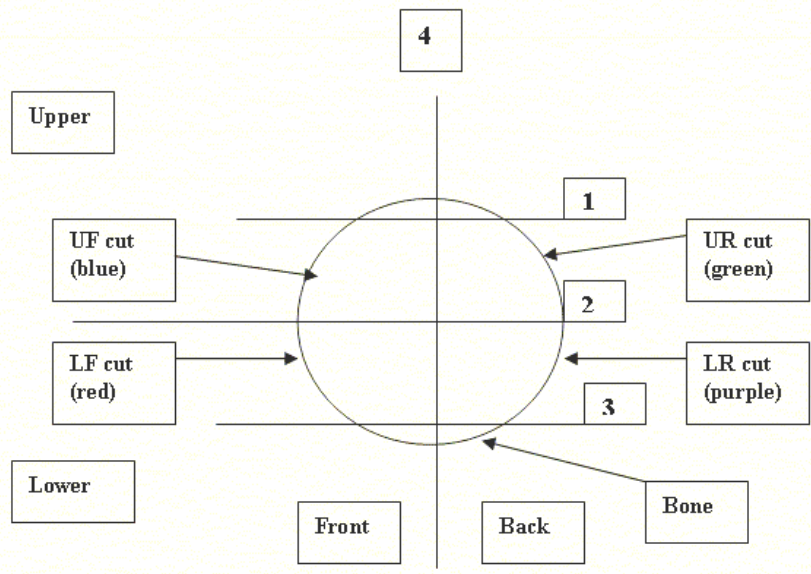

Figure 2: Bone section. Characterization of specimens according to their location in the bone.

\section{Materials and Methods}

\section{Experimental design}

Series of animal tests have been performed in-vitro on cuts of sheep femoral heads. Twelve femoral bones were tested with X-ray to ensure that the animal was sufficiently mature with epiphyseal plates closed. The bones were carefully excised in order to remove the effect of soft tissues on the measurements and treated with $2 \mathrm{~N}$ Hydrochloric acid for 4 hours in order to simulate the mineral deficiency observed in osteopenia. Each bone then was rinsed and dried in vacuum to remove excess water. Next each bone was cut in the trochanteric area in four practically rectangular quadrants as shown in Figure 1. From each bone four specimens were produced on which MDF, BMD, RS and IEF measurements were performed.

Axis 0 is the longitudinal axis of the bone. Levels 1,2 and 3 are the levels cutting the bone. These levels form an angle $120^{\circ}$ with the longitudinal axis of the bone. A fourth cutting level is needed in order to get four specimens from each bone. This fourth level is perpendicular to the three levels 1-3, as shown in Figure 1. Eventually, upper, lower, front, and back areas appear (Figures 1\&2). Additional cutting was performed in order to achieve rectangular specimens' shape. Thus, from each femur four integral specimens (cuts) were taken. The 45 cuts that were gathered from the 12 femora ( 3 cuts destroyed during cutting) were characterized according to their location in the bone, numbered and marked with blue, red, purple, and green color, as follows:

- $\quad$ UF cut (upper front) / blue

- $\quad$ LF cut (lower front) / red

- $\quad$ UR cut (upper rear) / green

- $\quad$ LR cut (lower rear) / purple

The above preparations and setup occurred as a result for:

- Elimination of any effect of surrounding soft tissue on MDF - QF measurements

- $\quad$ Specimens uniformity, achieved by cutting the trochanteric area in rectangular quadrants since most of the cortical bone is removed leaving rather homogeneous cancellous bone for testing 
- $\quad$ Better demineralization conditions using $\mathrm{HCl}$ acid and better specimen penetration

- Similar geometry of the specimen with practically same amount of bone material for testing.

Based on the methods described in the next sections, mass $\mathrm{m}$, damped natural frequency $\omega_{\mathrm{d}}$, MDF, BMD, RS and IEF measurements were performed on sheep femora cuts.

\section{Measurement of damping}

Natural frequency of any structural member is defined as the frequency at which the member vibrates if displaced from equilibrium. Usually several frequencies coexist and signal analysis techniques are available for the simultaneous measurement of these frequencies. Material damping factor $\gamma$ is defined as the energy dissipated throughout the medium in one cycle of deformation, normalized with respect to the elastic energy stored during that cycle, representing the fraction of strain energy lost in one full cycle [34] due to discontinuities of the medium.

The present study is motivated by the need to quantify material damping for the design of such structures. Knowledge of the fundamental sources of damping existing in all solids has been around for over fifty years [35-37]. Of these fundamental sources, thermoelastic dissipation is the most significant [29-31]. The modal damping factor $\zeta$ (MDF) and Quality Factor (QF) are defined $[34,38]$ as

$$
\begin{aligned}
& \zeta=\sqrt{\gamma^{2} /\left(4+\zeta^{2}\right)} \\
& Q F=\frac{1}{2 \zeta}
\end{aligned}
$$

From [22], eq/on (3.74), page 146

$$
2 \zeta / \sqrt{\left(1-\zeta^{2}\right)} \cong 2 \zeta=\left(\omega_{n} / \omega\right) \times \gamma
$$

Hence, for natural vibration $\left(\omega / \omega_{n}\right)=1$ and $(\delta / \pi)=\gamma \cong 2 \zeta$

$$
\gamma=2 \zeta
$$

Then, raising to the second power both sides of equation (1) we get

$$
\zeta^{2}=\gamma^{2} /\left(4+\zeta^{2}\right) \Rightarrow 4 \zeta^{2}+\zeta^{4}=\gamma^{2}
$$

From equations (4) and (5) we get $\zeta^{4}=0$, which is true, given that $\zeta$ is too small. Hence equation (1) is valid.

Dedicated device capable for MDF measurements was designed and constructed, consisting of a triggering unit which emits a sound transmitted to the bone through contact of a stem and of an accelerometer as output sensor. MDF was obtained in vitro by applying the half-power bandwidth method [34]. Bones with all soft tissues carefully removed were used. A piezoelectric accelerometer of $0,5 \mathrm{~g}$ mass was fixed on one end of the femur as vibration transducer. The bone was set to free vibration from an initial displaced position and the signal of the accelerometer was then amplified and relayed to an analogue-todigital converter. The vibration data were stored in computer for further analysis. MDF was calculated from the FFT analysis of time response data. The damping factor is proportional to the energy absorbed per cycle of vibration [34].

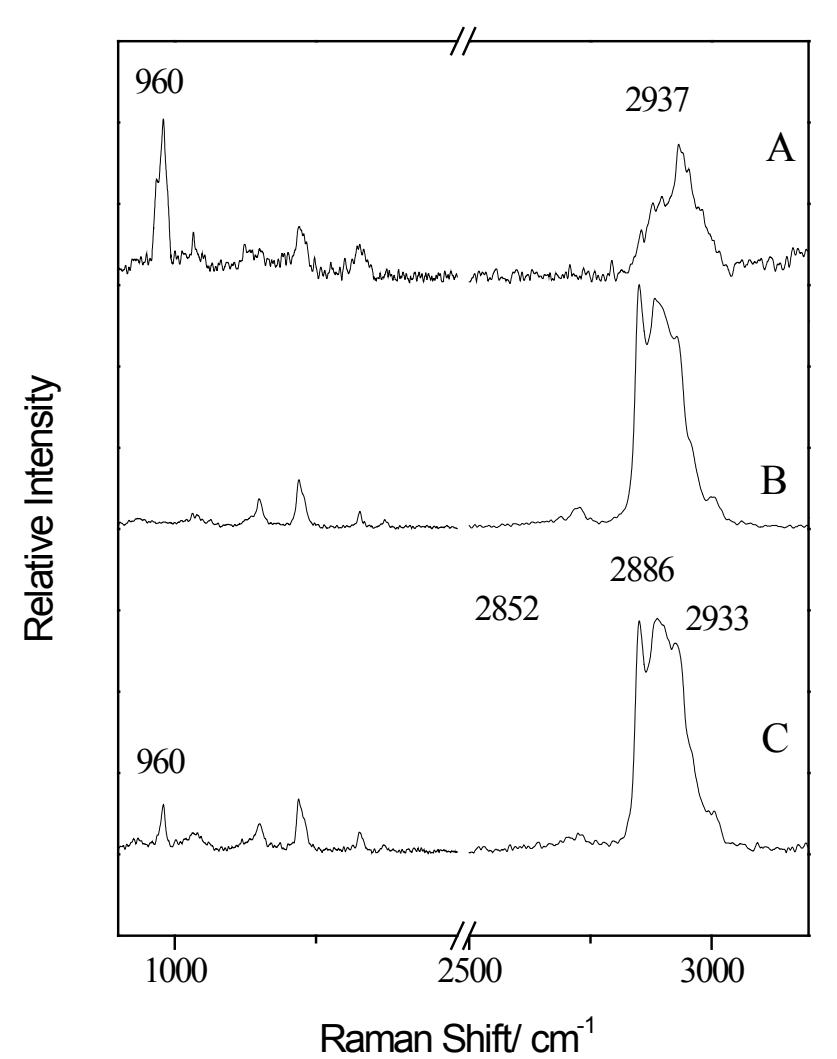

Figure 3: Typical Raman spectra of the cortical region of a bone.

\section{Measurement of BMD}

BMD was measured by Dual-Energy X-ray Absorptiometry (DEXA), using a Norland XR-26 MARK II bone densitometer. Scans were acquired and processed with an ultra-high resolution software program (available from the manufacturer). The measurements were performed on cuts taken from the neck of the femoral head. The time required for a typical scan at each site was approximately 3 minutes. This method was considered due to the fact that it is now recognized as the most important single determinant of fracture risk in osteoporotic populations.

\section{Measurement of RS}

Major advantage of the Raman technique is that the use of laser as excitation source permits the point-by-point analysis (mapping) of the sample. Raman spectra were recorded using a FRA-106/S FT-Raman (Bruker, Karlsruhe, Germany) with the following characteristics: Laser excitation line used was the $1064 \mathrm{~nm}$ of an Nd:YAG laser. The reference source (He-Ne laser) was used to measure the instrumental response and for checking of the interferometer. Filters were used to remove the Rayleigh line and the optical output of the He-Ne laser. The scattered light was collected at an angle of 1800 (back-scattering). The system was equipped with a LN2 cooled Ge detector (D 418). The power of the incident laser beam was about $150 \mathrm{~mW}$ on sample's surface. Typical spectral width was $2 \mathrm{~cm}^{-1}$. The system was interfaced with a computer. The diameter of laser beam's spot on the sample was $0.1 \mathrm{~mm}$. An X-Y-Z motor (Bruker, Karlsruhe, Germany) was used for "mapping" the sample.

Typical Raman spectra of the cortical region of a bone can be seen 


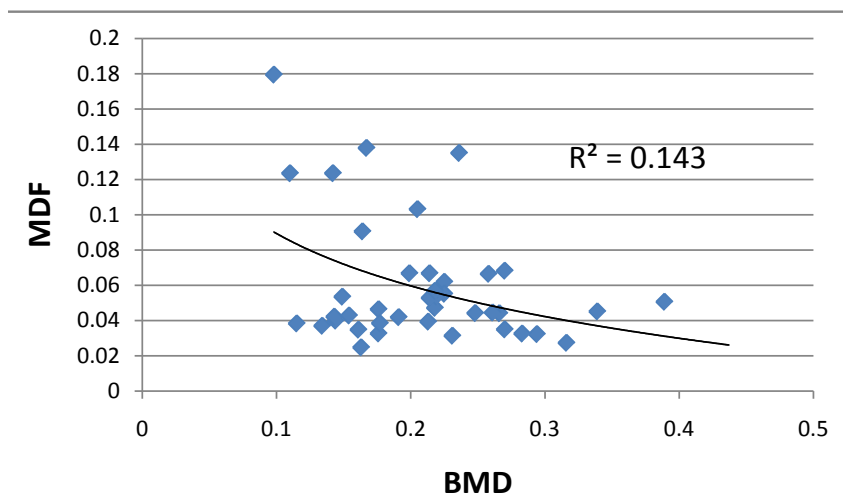

Figure 4: MDF vs BMD measurements on all cuts.

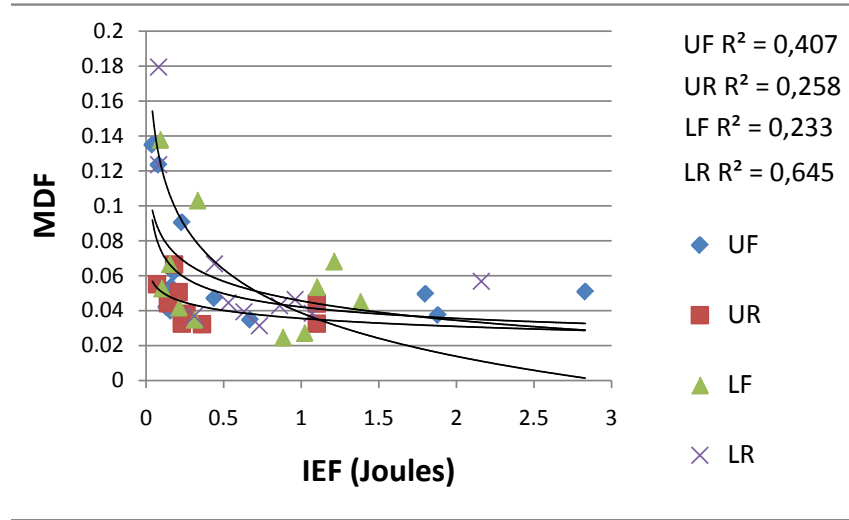

Figure 5: MDF vs IEF measurements on sheep trochanters cuts according to the location of the cut in the bone.

in Figure 3A. The strong symmetric vibration of bioapatite phosphoric group band can be seen at $960 \mathrm{~cm}^{-1}$ while the Raman intensity at 2937 $\mathrm{cm}^{-1}$ is attributed to the presence of bone collagen.

Raman spectra from the cancellous region of sheep cuts before and after the demineralizing effect of hydrochloric acid can be seen in Figures 3C and 3B, respectively. The organic spectral component changes considerably, with phospolipid spectrum dominating the 2800 $-3000 \mathrm{~cm}^{-1}$ region (2852 and $2886 \mathrm{~cm}^{-1}$ corresponding to symmetric and asymmetric $\mathrm{CH}_{2}$ stretches of lipids). The absence of the characteristic symmetric $v_{1}$ vibration of the phosphoric group at $960 \mathrm{~cm}^{-1}$ in Figure $3 \mathrm{~B}$ indicates a cancellous region with significant mineral deficiency as opposed to the cancellous region of Figure 3C. The remaining mineral in each cut after demineralization was determined as follows: Four Raman spectra from different spots of the spongy region of each cut before demineralization (control spectra) were recorded and the average of the four ratios, RC, of the $960 \mathrm{~cm}^{-1}$ Raman peak area, A960, to the area of the organic components $\left(2800-3000 \mathrm{~cm}^{-1}\right)$, Aorg, was calculated. This ratio was then compared to the respective ratios, $\mathrm{RD}$, of the artificially demineralized cuts. Assuming that the demineralized agent caused no significant change in the organic part of the spectra [10], the remaining mineral as percentage of the initial bioapatite present was calculated by dividing the ratios i.e. $\mathrm{RD} / \mathrm{RCx} 100=$ Remaining mineral $\%$.

\section{Measurement of IEF}

The IEF measurements were performed with the Pendulum Impact
Tester 53301, which is used for the determination of impact strength of specimens according to DIN 53453 and ISO R 179. The resistance to impact bending tests on notched and unnotched specimens or to impact tensile tests, as defined by applicable standards, is the impact energy absorbed by the specimen referred to the smallest cross - section of the specimen prior to the test. In order to obtain data about the mechanical capacity of the specimen product to withstand stresses, the measured value which is determined for a classification of the toughness and brittleness, as well as of the behaviour at a certain impact bending stress, is of fundamental significance. The bone specimens were broken by sudden impacts hitting the specimen. The KB Pendulum Impact Tester 53301 was used to determine the absorbed impact energy [39].

\section{Results}

The above methodology was applied in order to assess the possibility of using MDF as a monitoring tool of bone structural integrity. Our previous research findings led us to the belief that changes in MDF and QF due to the change in bone structure are correlating with bone density changes.

The relation between MDF - BMD measurements for the total number of cuts is presented in Figure 4.

From Figure 4 it is apparent that MDF increases with decreasing BMD. This situation corresponds to loss of minerals, hence decrease of bone density and degradation of bone structural integrity. This is something expected from vibrations theory due to thermo elastic effect, given that MDF expresses the dissipated energy due to structural defects, and takes values from 0 to 1 , where larger values correspond to structures with higher porosity and vice versa.

The MDF - IEF correlation per cut is presented in Figure 5, while the correlation of MDF - IEF measurements for all tested specimens, regardless of their location in the trochanter, are presented in Figure 6.

From both Figures 5\&6, it can be seen that when bone porosity increases, something that corresponds to increasing MDF, the energy needed for fracture of the bone is decreasing (IEF), something expected for degradated bones of decreased strength.

The rational of using MDF measurements can be also seen in Figure 7, where the remaining mineral percentage, that was calculated from Raman spectra recorded from the cancellous region of each cut exposed to laser Raman excitation after the application of IEF to each of the four cuts for each bone, correlates in an inverse way to MDF, i.e.

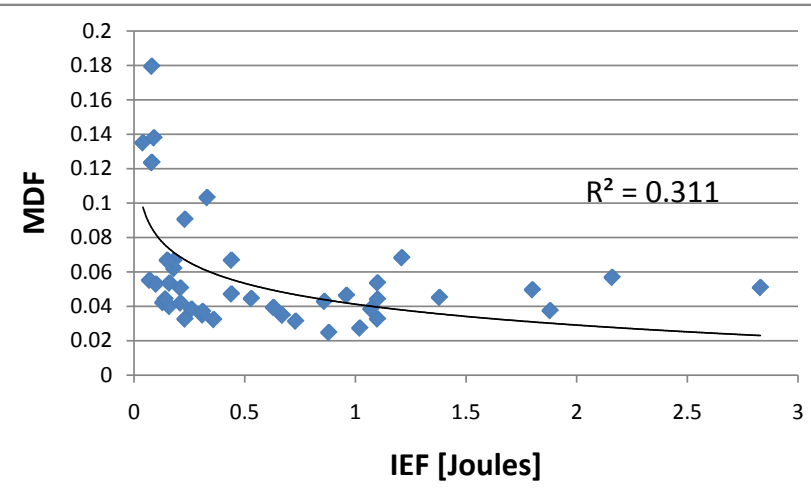

Figure 6: MDF vs IEF measurements on sheep trochanters cuts. Global correlation for all cuts. 
Citation: Panteliou SD, Panagiotopoulos E, Varakis J, Orkoula M, Kontoyannis C (2012) Measurement of Modal Damping Factor to Monitor Bone Integrity and Osteopenia: A Study on Sheep Trochanters. J Bioengineer \& Biomedical Sci 2:118. doi:10.4172/2155-9538.1000118

Page 5 of 6

the presence of more bioapatite which expresses bone of better quality results to lower MDF values and vice versa as expected.

Taking into account that changes in IEF can give valuable information about bone's absolute strength values, comparative to IEF measurements presentations of the results obtained with BMD and Remaining Mineral (RS) are shown in Figures $8 \& 9$ respectively. Although these results [39] refer to material properties and do not take into account the moment of inertia related to bone geometry, and the energy absorbing mechanisms, they are better indicators of bone resistance to fracture, as they are closer to fall mechanics (impact injury).

\section{Discussion}

On the basis of the above findings it is apparent that the proposed methodology, when used as a bone condition-monitoring tool, can give valuable information for the structural integrity of the bone. More precisely, as stated in previous authors' works [11,27,28, 40,41], MDF accounts directly for changes in bone architecture. It has been shown that MDF or QF, are directly related with stress concentration due to structural changes [29], such as the change in bone porosity accompanying osteopenia. Besides, stress concentration is known to cause fracture in materials with such discontinuities.

From the animal studies conducted in this work it is shown that MDF or QF are indicators and measures of the tendency of

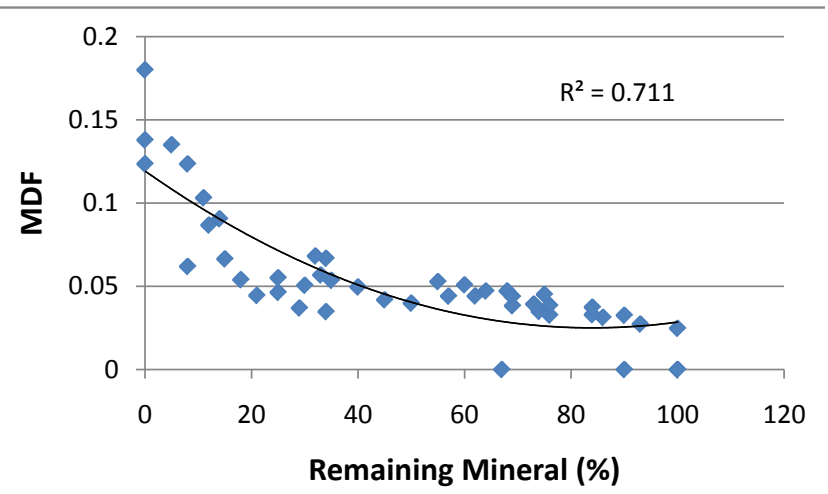

Figure 7: MDF vs the percentage of the Remaining Mineral calculated from Raman Spectroscopy data on all cuts.

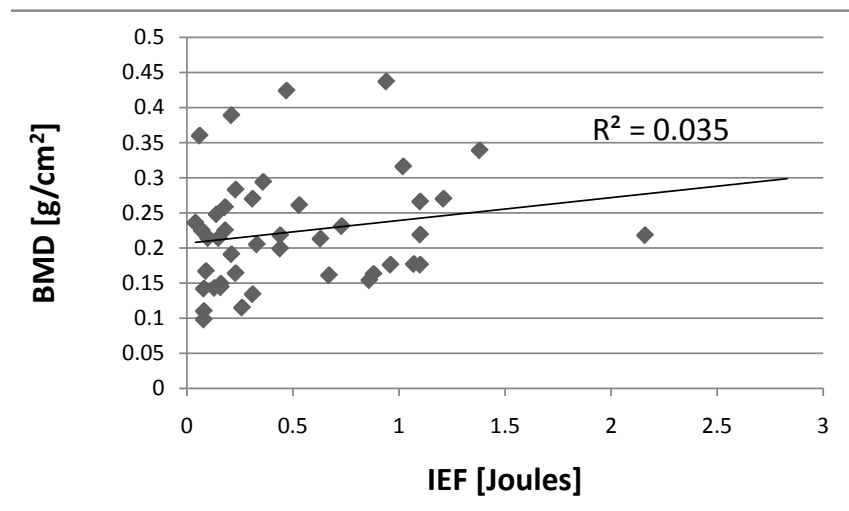

Figure 8: BMD vs IEF measurements on all cuts.

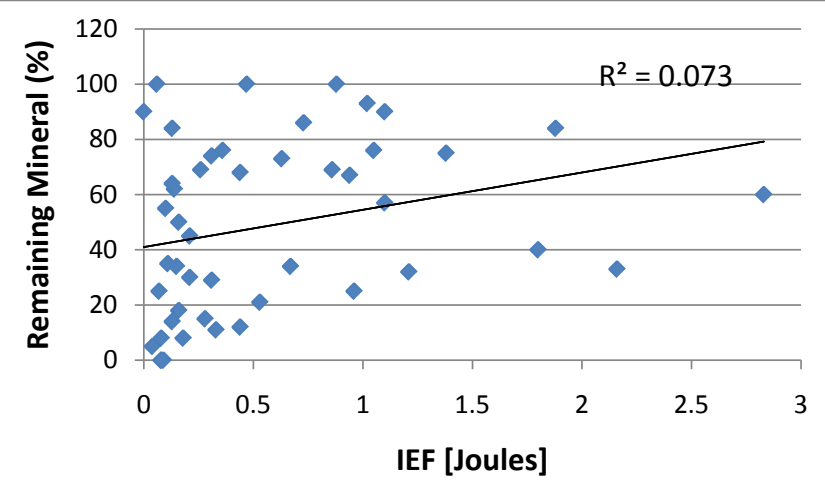

Figure 9: Remaining Mineral vs IEF measurements on all cuts.

the deteriorated bone to fracture, and correlate satisfactorily to the conventional methods (BMD, RS) and to the absolute strength measure (IEF).

The conventional methods for bone quality measurement have the following disadvantages: subjectivity, high cost, patient radiation, invasiveness, non portability, require trained personnel, disease must have started in order to be detected. On the contrary, MDF method has the following advantages: objectivity, higher accuracy and sensitivity than every known up to now method for assessment of bone density, repeatability, very low cost in comparison to that of the machines used for bone density measurement, non invasiveness, absence of pain, portability, no patient radiation hence method can be applied whenever wanted, short measurement duration (2-3 min), simple data acquisition procedure without demanding highly trained personnel, disease detection much earlier than any other method. Besides, according to our previous findings [28], the method is insensitive to variations in soft tissues composition and MDF results correlate with results acquired with all conventional methods, something that does not happen among them. Finally, MDF data can be teletransfered for diagnosis in central medical units of patients living in remote areas.

All above are in support of the effectiveness of the method when applied in a localized scale and reinforce its potential to become the diagnostic tool for monitoring bone structural changes related to metabolic bone diseases in clinical practice.

\section{References}

1. Mundy GR (1995) Bone remodelling and its disorders. (2ndedn), Martin Dunitz Itd, London.

2. Melton LJ, Cooper C (1998) Osteoporosis. J. Stevenson and R. Lindsay, New York.

3. Martin T J, Dempster D W, (1998), Osteoporosis. J. Stevenson and R. Lindsay New York.

4. Penel G, Leroy G, Bres E (1998) New preparation method of bone samples for Raman microspectrometry. Applied Spectroscopy, 52: 312-313.

5. Penel G, Leroy G, Rey C, Bres E (1998) MicroRaman spectral study of the PO4 and $\mathrm{CO} 3$ vibrational modes in synthetic and biological apatites. Calcif Tissue Int 63: 475-481.

6. Rehman I, Smith R, Hench L, Bonfield W (1995) Structural evaluation of human and sheep bone and comparison with synthetic hydroxyapatite by FT-Raman spectroscopy. J Biomedical Mater Res 29: 1287-1294.

7. Sauer GR, Zunic WB, Durig JR, Wuthier RE (1994) Fourier Transform Raman spectroscopy of synthetic and biological Calcium Phosphates. Calcif Tissue Int 54: 414-420. 
Citation: Panteliou SD, Panagiotopoulos E, Varakis J, Orkoula M, Kontoyannis C (2012) Measurement of Modal Damping Factor to Monitor Bone Integrity and Osteopenia: A Study on Sheep Trochanters. J Bioengineer \& Biomedical Sci 2:118. doi:10.4172/2155-9538.1000118

8. Walters WA, Leung YC, Blumenthal NC, LeGeros RZ, Konsker KA (1990) A Raman and Infrared spectroscopic investigation of biological Hydroxyapatite. J Inorg Biochem 39: 193-200.

9. Timlin JA, Carden A, Morris MD (1999) Chemical microstructure of cortical bone probed by Raman transects. Applied Spectroscopy 53: 1429-1435.

10. Kontoyannis CG, Vagenas NV (2000) FT-Raman spectroscopy: a tool for monitoring the demineralization of bones. Journal of Applied Spectroscopy 54 1605-1609.

11. Panteliou SD, Xirafaki AL, Panagiotopoulos E, Varakis JN, Vagenas NV, et al. (2004) Modal damping for monitoring bone integrity and osteoporosis. J Biomech Eng 126: 1-5.

12. Morris MD, Finney WF (2004) Recent developments in Raman and infrared spectroscopy and imaging of bone tissue. Spectroscopy: An International Journal 18: 155-159

13. Cumings SR, Black DM, Nevitt MC, Browner W, Cauley JA, et al. (1993) Bone density at various sites for prediction of hip fractures. Lancet 341: 72-75.

14. Cumings SR, Black DM, Nevitt MC, Browner W, Cauley JA, et al, (1990) Appendicular bone density and age predict hip fracture in women. The study of osteoporotic fractures research group. JAMA 263: 665-668.

15. Cumings SR, Nevit MC, Browner WS, Stone K, Fox KM, et al. (1995) Risk factors for hip fracture in white women. Study of osteoporotic fractures research group. N Engl J Med 332: 767-773.

16. Parfitt AM, Drezner MK, Glorieux FH, Kanis JA, Malluche H, et al. (1987) Bone histomorphometry: standardization of nomenclature, symbols, and units. Report of the ASBMR Histomorphometry nomenclature committee. J Bone Miner Res 2: 595-610.

17. Kleerekoper M, Villanueva AR, Stanciu J, Sudhaker RD, Parfitt AM (1985) The role of three-dimensional trabecular microstructure in the pathogenesis of vertebral compression fractures. Calcified Tissue International 37: 594-597.

18. Petersen K (1977) Noninvasive determination of bone stiffness. Dissertation, Stanford University.

19. Steele CR (1984) Instruments for assessment of bone properties. Workshop on Advances in NASA-Relevant Minimally Invasive Instrumentation, Asilomar, Ca.

20. Steele CR, Zhou LJ, Guido D, Marcus R, Heinrichs WL, et al. (1988) Noninvasive determination of ulnar stiffness from mechanical response-in vivo comparison of stiffness and bone mineral content in humans. J Biomech Eng 110: 87-96.

21. Doemland HH, Stanley B (1979) A method monitoring the healing of human long bones. IEEE /Eng in Med and Biol.

22. Hiriyama T, Sekiguchi T (1979) Assessment of fracture healing by vibration Acta Orthop Scand 50: 391-398.

23. Lewis JL (1975) A dynamic model for a healing fractured long bone. Journal of Biomech 8: 17-25.

24. Markey EL, Jurist JM (1974) Tibial resonant frequency measurement as an index of the strength of Fracture. Wis Med J 73: S62-65.

25. Campbell JN, Jurist JM (1971) Mechanical impedance of the femur: a preliminary report. J Biomech 4: 319-322.

26. Heaney RP, Avioli LV, Chesnut CH, Lappe J, Recke RR, et al. (1989) Osteoporotic bone fragility. Detection by ultrasound transmission velocity. JAMA 262: 2986-2990

27. Panteliou SD, Abbasi-Jahromi H, Dimarogonas A, Kohrt W, Civitelli R (1999) Low-frequency acoustic sweep of bone integrity and osteoporosis. J Biomech Eng 121: 423-431.

28. Anastassopoulos G, Panteliou SD, Christopoulou G, Stavropoulou A, Panagiotopoulos E, et al. (2010) Vibrational bone characteristics versus bone density for the assessment of osteoporosis in ovariectomized rats. J Med Eng Technol 34: 35-42.

29. Panteliou SD, Dimarogonas AD, Dimarogonas AD (1997) Thermodynamic damping in porous materials with ellipsoidal cavities. Journal of Sound and Vibration 201: 555-565.

30. Panteliou SD, Dimarogonas AD, Dimarogonas AD (1997) Thermodynamic damping in porous materials with spherical cavities. Journal of Shock and Vibration 4: 261-268.
31. Panteliou SD, Dimarogonas AD (2000) Damping associated with porosity and crack in solids. Theoretical and Applied Fracture Mechanics 34: 217-223.

32. Panteliou SD, Chondros T, Argyrakis V, Dimarogonas A (2001) Damping factor as an indicator of crack severity. Journal of Sound and Vibration, 241: 235-245.

33. Panteliou SD, Zonios K, Chondrou IT, Fernandes HR, Agathopoulos S, Ferreira JMF (2009) Damping factor associated with porosity in alumina. Int J Mech Mat Des 5: 167-174.

34. Dimarogonas AD (1996) Vibration for Engineers. (2nd edition), Englewood Cliffs, New Jersey, USA.

35. Silver MJ, Peterson DL, Erwin SR (2002) Predictive thermoelastic damping in beams using finite element techniques. American Institute of Aeronautics and Astronautics: 1729

36. Braginsky VB, Mitrofanov VP, Panov VI (1985) Systems with small dissipation University of Chicago Press, Chicago IL, USA.

37. Zener C (1948) Elasticity and anelasticity of metals. The University of Chicago Press, Chicago IL.

38. Lazan BJ (1968) Damping of materials and members in structural mechanics Pergamon Press, Oxford, UK

39. Panagiotopoulos E, Kostopoulos V, Tsantzalis S, Fortis AP Doulalas A (2005) Impact energy absorption by specimens from the upper end of the human femur. Injury 36: 613-617.

40. Stavropoulou A, Christopoulou GE, Anastassopoulos G, Panteliou SD, Lyritis GP, et al. (2005) Alteration in serum leptin correlates with alterations in serum $\mathrm{N}$-telopeptide of collagen type I and serim osteocalcin during the progression of osteoporosis in ovariectomized rats. Clin Chem Lab Med 43: 1359-1365.

41. Christopoulou GE, Stavropoulou A, Anastassopoulos G, Panteliou SD, Papadaki E, et al. (2006) Evaluation of modal damping factor as a diagnostic tool for osteoporosis and its relation with serum osteocalcin and collagen $\mathrm{N}$-telopeptide for monitoring the efficacy of aledronate in ovariectomized rats. $J$ Pharm Biomed Anal 41: 891-897. 